\title{
ARTICLE
}

\section{PANTHERE: simulation software for 3D dose rate calculation in complex nuclear facilities}

\author{
Matthieu Longeot $^{\mathrm{a}^{*}}$, Bruno Dupont ${ }^{\mathrm{a}}$, Mark Zweers ${ }^{\mathrm{b}}$, Fausto Malvagi ${ }^{\mathrm{c}}$, Jean-Christophe Trama ${ }^{\mathrm{c}}$ and Julien Dubost ${ }^{\mathrm{d}}$ \\ ${ }^{a}$ Electricité de France (EDF), SEPTEN/TE/RP, 12-14 avenue Dutriévoz, 69628 Villeurbanne, France; ${ }^{b}$ EDF/R\&D/SINETICS, \\ 1 avenue du Général de Gaulle, 92141 Clamart, France; ${ }^{~}{ }^{C}$ Commissariat à l'Energie Atomique/Saclay DANS/DM2S/SERMA, \\ 91191 Gif-sur-Yvette, France; ${ }^{d}$ CS SI, 45 rue Sainte Geneviève, 69006 Lyon, France
}

\begin{abstract}
PANTHERE is an industrial simulation tool developed by EDF to calculate $\gamma$ dose rates in 3D geometry with numerous observation points and radioactive sources. It is composed of three main components: a kernel, a GUI and a database. PANTHERE takes a key role in EDF's ALARA process, enabling to predict dose rates and then organize and optimize operations in high radiation environments. It is used in most EDF engineering units at every stage of a nuclear facility lifecycle: design (EPR ${ }^{\mathrm{TM}}$, for example), operation (maintenance) and dismantling. PANTHERE is verified and validated by comparison with reference Monte-Carlo code TRIPOLI-4 ${ }^{\circledR}$ and gamma dose rates experimental measurements. EDF is currently replacing PANTHEREV1 (operational version) by PANTHEREV2. The major improvements are the enhanced computation speed, the unification of the GUI and the shared database for all radiation protection simulation codes used by EDF engineering units (gamma, and in the future beta and neutron radiation). PANTHEREV2's kernel uses the NARMER solver of the CEA, which performs dose rate calculations based on the point-kernel integration method with build-up factors. The GUI is based on the software platform SALOME® of CEA and EDF, and provides improved ergonomy and contains all necessary features as required by radiation protection simulation practices.
\end{abstract}

Keywords: point kernel method; radiation protection; dosimetry; graphical user interface; modeling; CAD

\section{Introduction}

PANTHERE (acronym for Predictions ANd THeoretical analyses of Exposure in REactors) is a radiation protection code used for $3 \mathrm{D}$ simulation of gamma dose rates and fluence in complex nuclear facility with distributed radioactive sources. It stands between powerful but complex radiation protection computer codes (like CEA Monte Carlo code TRIPOLI-4® [1]) and simplified tools dedicated to simple geometries (like MICROSHIELD $\AA$ ). Therefore it is the industrial solution adapted to radiation simulation in complex nuclear facilities

PANTHERE is intensively used by EDF engineering units and occasionally in the CEA and AREVA-NC. It was originally used mainly to prepare maintenance and modification operations in nuclear power plants, but it is now extended to other applications like:

a) shield designs and zoning for: $\mathrm{EPR}^{\mathrm{TM}}$ reactors (France, UK); nuclear waste storage facilities;

b) integrated dose calculations to qualify PWR equipments during a severe accident;

c) decontamination and dismantling operations (dosimetry prediction and optimization); d) radiological characterization notably for applications associated to transport and nuclear waste management.

As for the context of usage, several tools are related to PANTHERE: a) software and method to predict nuclide activities (fuel cycle simulation), or devices to measure them (gamma spectroscopy, for example); b) Computer Aided Design software (Solidworks ${ }^{\mathrm{TM}}$ or PDMS $^{\mathrm{TM}}$ ) with the possibility of CAD transfer to PANTHERE.

Finally, PANTHERE is verified and validated for generic usage in EDF engineering units. PANTHERE is acknowledged by the French Nuclear Safety Authority as the reference code for the sizing of biological protections within the EPR ${ }^{\mathrm{TM}}$ buildings.

\section{The physical models used}

PANTHERE's computation kernel uses the NARMER solver [2] of the CEA, which is linked to the geometric pursuit library LIBCAD of EDF/R\&D; both are developed in the $\mathrm{C}++$ language. This chapter focuses on the different physical models used by NARMER.

*Corresponding author. Email: matthieu.longeot@edf.fr 


\subsection{The point kernel integration method}

The method used in NARMER is the point-kernel integration method with build-up factors. It is a macroscopic approach for gamma dose rate calculation, taking into account the interaction with matter. For example, the Dose Equivalent Rate (DER) for a mono-energetic source of activity $A\left(\mathrm{gamma} /\left(\mathrm{cm}^{3} . \mathrm{s}\right)\right)$ in a volume Vs is computed as in Eq. (1).

$\operatorname{DER}(E)=k(E) * \iiint_{\mathrm{Vs}} B U(E, \mu(E) \mathrm{x}) * \frac{e^{-\mu(E) x}}{4 \pi d^{2}} * A(E) * d V$

with:

- $k(E)$ the conversion factor from gamma flux to dose rate taken from the International Commission on Radiological Protection (ICRP) [3];

- $\mu(E)$ the macroscopic linear attenuation factor;

$-x$ the shield thickness encountered by $\gamma$ ray along a

straight line.

- $d$ the distance between point and source.

In the above formula, the exponential part represents the attenuation in the shielding material and the denominator the geometric attenuation.

\subsection{The build-up factors}

The build-up factors (BU in Eq. (1)) are used to estimate the scattered radiation in matter. These factors are tabulated for all simple elements (from $Z=1$ to 98) and for 195 energy groups between $15 \mathrm{keV}$ and $10 \mathrm{MeV}$. For multi-layer shields, the BU factors used in Eq.(1) are computed according to the "CEA/SERMA" formula [2].

\section{The PANTHERE architecture}

PANTHERE is constituted of three main components: a computational kernel, a MySQL database for administration and sharing of the models, and a 3D GUI to define the studies and the radiological environment. It runs on a linux operating system.

\subsection{Pretreatment in the GUI}

The PANTHERE GUI allows the definition of the geometrical and radiological models by the use of 10 parametrical primitives (e.g., cylinder, wall), frequently used to model nuclear facilities. They can be composed of 1 to 5 distinct media: 2 internal media (air/water) and 3 successive layers "metal", "thermal insulation" and "biological protection".

Radioactive sources can be added to every layer of each object (both volume and surface sources). The spectrum is defined with energy rays, with nuclides or using energy groups (195 groups, for example). The nuclear data are taken from JEF2.2 [4]. Activities in Bq can either be entered per $\mathrm{cm}^{3}$, per $\mathrm{cm}^{2}$ or per grams.

Observation points are defined by their 3D coordinates. Measurement results can be added to each observation point to perform a readjustment of the predefined source activity (fitting).

Finally, results can be given according to several response functions: the equivalent dose rate $H^{*}(10)$ in $\mathrm{mSv} / \mathrm{h}$, the "kerma in air" in $\mathrm{mGy} / \mathrm{h}$, the energetic fluence rate in $\mathrm{Mev} /\left(\mathrm{cm}^{2} . \mathrm{s}\right)$ and the uncollided flux (in gamma/( $\left.\left.\mathrm{cm}^{2} . \mathrm{s}\right)\right)$.

Figure 1 shows the PANTHEREV2 geometric model of a PWR primary loop.

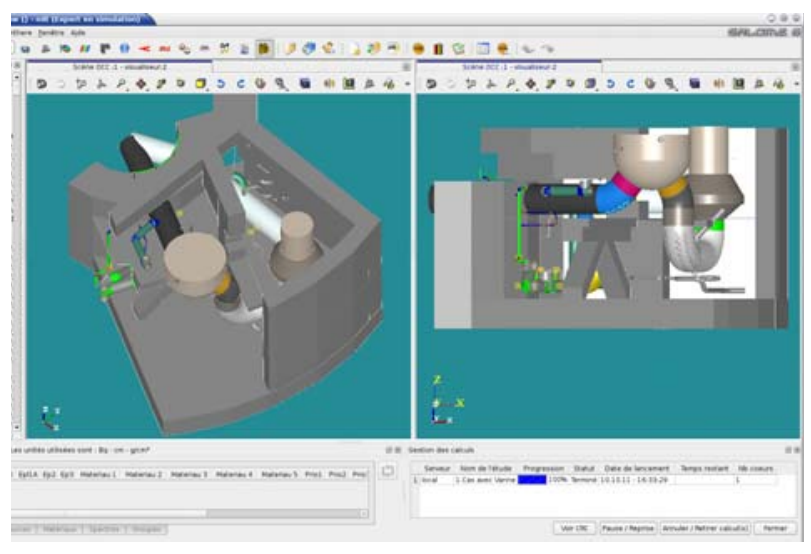

Figure 1. A primary loop of a PWR.

\subsection{Postprocessing in the GUI}

The results of the computation are shown as a source/point or nuclide/point array and can be exported in .csv format. Dedicated SALOME® (http://www.salome-platform.org) modules allow for radiological zoning and isodose mapping (cf. Figure 2).

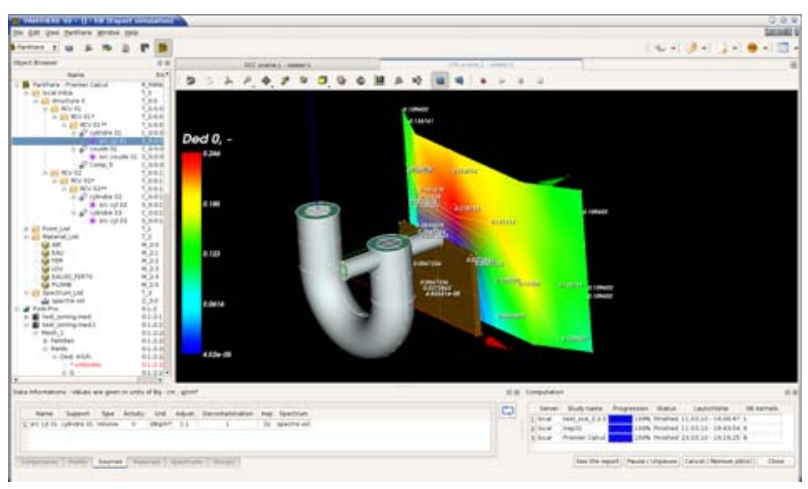

Figure 2. Zoning functionality in PANTHEREV2.

To complete the ALARA (As Low As Reasonably Achievable) principle, the simulation results of PANTHERE and maintenance task characteristics can be used as input of an EDF Decision-Making Aid Tool. This tool performs optimization of the collective dosimetry by scheduling the tasks in order to reduce the integrated dose rate of workers.

\section{V\&V of PANTHERE - domain of validity}

\subsection{Methodology of PANTHERE V\&V and illustration}

EDF/SEPTEN performs the validation of PANTHERE after analysis of verification performed by $\mathrm{C}-\mathrm{S}$ (software integrator) [5]. The objective is to define a validation domain focusing on industrial studies found 
in EDF engineering units. The V\&V of PANTHERE is based on benchmarks with international experimental results. Moreover, the calculation method bias is estimated by means of code-to-code benchmarks with the reference Monte-Carlo code TRIPOLI-4® (solving exactly the Boltzmann transport equation).

The "slant penetration experiment" [9] is one of the benchmarks used for the V\&V of PANTHEREV2. It consists of 10 detector points positioned behind a wall with slant angles between $0^{\circ}$ and $78^{\circ}$. Table 1 shows the results of TRIPOLI4 and PANTHEREV2 calculations performed by CEA and EDF as well as the ratio between PANTHEREV2 and measurement results. It demonstrates that for slant angles greater than appr. $73^{\circ}$, PANTHEREV2 underestimates the radiation exposure compared to experimental data.

Table 1. Comparison of computation results (TRIPOLI4, PANTHEREV2) with measurement results for the "slant penetration benchmark".

\begin{tabular}{|c|c|c|c|c|c|c|c|}
\hline $\begin{array}{c}\text { Slant } \\
\text { angle }(\boldsymbol{}(\boldsymbol{})\end{array}$ & $\begin{array}{c}\text { Measure } \\
(\boldsymbol{m} \boldsymbol{R} / \boldsymbol{h})\end{array}$ & $\begin{array}{c}\text { TRIPOLI4 } \\
(\boldsymbol{m} \boldsymbol{R} / \boldsymbol{h})\end{array}$ & $\begin{array}{c}\text { FSD } \\
(\%)\end{array}$ & $\begin{array}{c}\text { MCNP } \\
(\mathbf{m} \mathbf{R} \mathbf{h})\end{array}$ & $\begin{array}{c}\text { FSD } \\
(\%)\end{array}$ & $\begin{array}{c}\text { PANTHEREV2 } \\
(\boldsymbol{m} \boldsymbol{R} / \boldsymbol{h})\end{array}$ & $\begin{array}{c}\text { PV2/m easure } \\
(\%)\end{array}$ \\
\hline 0 & $1,24 \mathrm{E}+03$ & $1,43 \mathrm{E}+03$ & 0,23 & $1,37 \mathrm{E}+03$ & 3,42 & $1,35 \mathrm{E}+03$ & $9 \%$ \\
\hline 25 & $9,38 \mathrm{E}+02$ & $9,63 \mathrm{E}+02$ & 0,26 & $9,78 \mathrm{E}+02$ & 3,85 & $9,50 \mathrm{E}+02$ & $1 \%$ \\
\hline 43 & $3,85 \mathrm{E}+02$ & $3,77 \mathrm{E}+02$ & 0,40 & $3,64 \mathrm{E}+02$ & 4,09 & $4,03 \mathrm{E}+02$ & $5 \%$ \\
\hline 54 & $1,16 \mathrm{E}+02$ & $1,16 \mathrm{E}+02$ & 0,66 & $1,27 \mathrm{E}+02$ & 2,24 & $1,38 \mathrm{E}+02$ & $19 \%$ \\
\hline 62 & $3,55 \mathrm{E}+01$ & $3,38 \mathrm{E}+01$ & 1,06 & $3,93 \mathrm{E}+01$ & 3,27 & $4,28 \mathrm{E}+01$ & $21 \%$ \\
\hline 67 & $1,10 \mathrm{E}+01$ & $1,02 \mathrm{E}+01$ & 2,18 & $1,09 \mathrm{E}+01$ & 4,54 & $1,28 \mathrm{E}+01$ & $16 \%$ \\
\hline 70 & $3,52 \mathrm{E}+00$ & $2,86 \mathrm{E}+00$ & 2,77 & $3,56 \mathrm{E}+00$ & 4,94 & $3,83 \mathrm{E}+00$ & $9 \%$ \\
\hline 73 & $1,23 \mathrm{E}+00$ & $1,09 \mathrm{E}+00$ & 5,51 & $1,27 \mathrm{E}+00$ & 4,22 & $1,17 \mathrm{E}+00$ & $-5 \%$ \\
\hline 77 & $2,58 \mathrm{E}-01$ & $1,89 \mathrm{E}-01$ & 7,63 & $2,46 \mathrm{E}-01$ & 3,02 & $1,01 \mathrm{E}-01$ & $-61 \%$ \\
\hline 78 & $1,20 \mathrm{E}-01$ & $9,92 \mathrm{E}-02$ & 9,34 & $1,54 \mathrm{E}-01$ & 4,32 & $3,18 \mathrm{E}-02$ & $-74 \%$ \\
\hline
\end{tabular}

\subsection{Limitations of the physical model}

PANTHERE uses a simplified physical model to enhance calculation performance (10 to 25 times faster than stochastic methods like TRIPOLI-4®). However, it restricts the usage of PANTHERE to domains where the approximations of Eq. (1) are pertinent.

The main restrictions are geometrical: the singularity of Eq. (1) imposes a minimum distance between source and observation point and the straight-line approximation imposes a regular geometry between source and observation point.

Another point to keep in mind is that the current version of PANTHERE only takes into account direct gamma radiation. For instance, in a closed room, reflection by walls, roof and floor can significantly contribute to the dose rate, and has to be estimated using another method or code like Chilton-Huddleston albedo method [6] or Monte-Carlo code TRIPOLI-4®.

Finally, the build-up factors are validated for penetration lengths up to $50 \mathrm{MFP}$ [2], and for energies ranging from $15 \mathrm{keV}$ to $10 \mathrm{MeV}$. They should not be used outside this domain of validation.

Fortunately, this domain covers most of the studies for nuclear energy facilities.

\section{Improvements and actualization of nuclear data: PANTHEREV2}

PANTHERE has been used for approximately 15 years at EDF to evaluate dose rates, and even if the nuclear data have been updated, the application (GUI and the kernel) needed a "reactualization" in terms of ergonomy, performance and code maintainability. The non-regression of PANTHEREV2 with respect to V1 has been proven for elementary and industrial test cases. The first operational version of PANTHEREV2 will be released soon. The developments performed in the last five years have produced efficient, modern and operational software to estimate dose rates, using nuclear data from JEF2.2.

PANTHEREV2 contains improvements in terms of data management to better respond to industrial process requirements.

\subsection{Ergonomy}

PANTHEREV2 provides substantial improvements compared to V1 in terms of GUI ergonomy. The organization of the different panels has been reconsidered, and a lot of classical keyboard and mouse shortcuts have been added to improve the user efficiency.

\subsection{Improved import of CAD models in PANTHERE}

PANTHERE inherits SALOME's CAD modeler (GEOM) which allows designing buildings, structures and piping. Moreover, PANTHEREV2 allows to import CAD data from specific modelers like Solidworks ${ }^{\mathrm{TM}}$ or

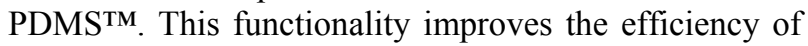
the geometric modeling and allows to model complex nuclear facility with better resemblance. Figure 3 gives an example of the types of external CAD models that can be imported in PANTHEREV2.

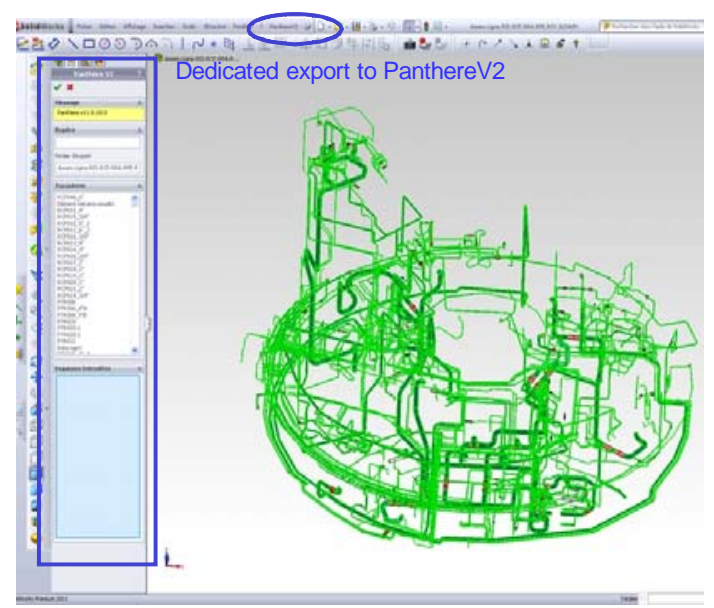

Figure 3. PWR piping systems (with metadata) designed with Solidworks ${ }^{\mathrm{TM}}$, to be transferred to PANTHEREV2.

\subsection{Computational performance}

The kernel of PANTHEREV2 improves computational performance by using an optimized 
geometrical representation and raytracer, and by implementing shared-memory parallelization (using Intel TBB (Threading Building Blocks) over the observation points.

Further acceleration could be obtained by distributed computing currently under development.

\section{PANTHERE usage in EDF engineering units}

This paragraph describes the application of PANTHERE to the optimization of radiation protection in preparation of a maintenance operation in a nuclear power plant. It is given here as an illustration to situate PANTHERE calculations in the ALARA process. The diagram in Figure 4 macroscopically presents the methodology used for the optimization of the dosimetry for a work in high radiation environment.

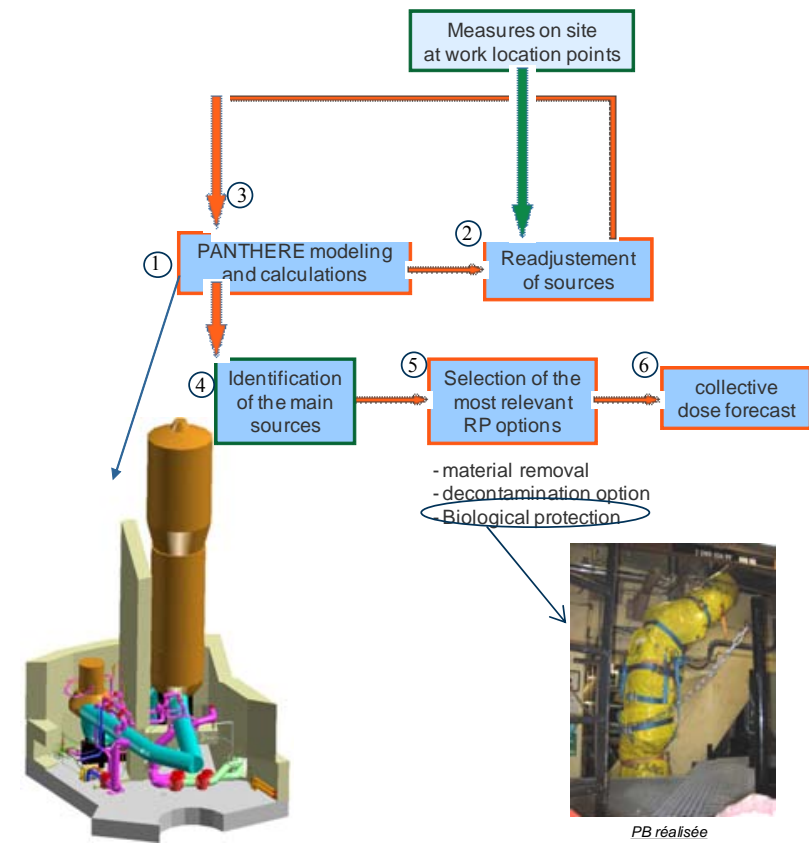

Figure 4. Methodology used for optimization of the dosimetry for a maintenance operation on a PWR primary loop.

At the beginning, PANTHERE calculations are performed with pre-defined sources (estimated from field experience) to generate a transfer function from source activity to DER. By means of measurements performed on-site at work location points, source activities can be fitted to reality, and give the appropriate DER. Finally, radiation protection options can be selected in order to lower the collective dose for the task.

Note that in Figure 4, the collective dose for a given task is defined as follows (see [7]):

with:

$$
C D=D E R^{*} \text { Work volume } K
$$

- CD: Collective Dose in [Man.Sv];

- DER: Dose Equivalent Rate in [Sv/unit of time];

- Work volume: in [Man.unit of time];
- K: exposure coefficient and/or intervention coefficient (ergonomy) etc.

\section{Conclusion}

PANTHERE plays an important role in an ALARA regulatory process by providing simulation results for industrial radiation protection. Its use of a simplified computation method and its user-friendly GUI dedicated to radiation protection allow a reliable and fast industrial usage to maintain occupational radiation exposures As Low As Reasonably Achievable in a nuclear facility.

PANTHEREV2, with an improved GUI and enhanced computational performance, will be released soon. This version will enhance productivity and quality. It will benefit from ongoing developments by EDF R\&D to increase performance and transfer complex CAD models from external modelers. Moreover, future releases will include the computation of beta (short-term) and neutron (long-term) dose rates.

\section{References}

[1] TRIPOLI-4 Project Team, Tripoli-4 User Guide, Report CEA-R-6169, Commissariat à l'Energie Atomique, (2008).

[2] C. Suteau, M. Chiron and G. Arnaud, Improvement of MERCURE-6's General Formalism for Calculating Gamma-Ray Buildup Factors in Multilayer Shields, Nucl. Sci. \& Eng. 147 (2004), pp. 419-424.

[3] ICRP (International Commission on Radiological Protection) 74 (1995); Conversion Coefficients for use in Radiological Protection against External Radiation.

[4] The JEF-2.2 Nuclear Data Library, NEA JEFF Report 17 (2000).

[5] M. Longeot, B. Dupont, C. Coatanea, A. Schumm, M. Zweers, F. Malvagi and J.-C. Trama, PANTHERE: Simulation software for dose rate calculation in complex nuclear facility, Proc. 4th Francophone Days on Rad. Prot. Calc. Codes (SFRP), Sochaux, France (2010). [in French]

[6] A. Chilton and C. Huddleston, A semi-empirical formula for differential dose albedo for gamma rays on concrete, Nucl. Sci. \& Eng. 17 (1963), pp. 419-424.

[7] A. Brissaud, E. Peluso, P. Ridoux and T. Tribollet, PANTHEREV1: a tool for dose rate calculation, Proc. Int. ALARA Symposium, Orlando, USA, (1999).

[8] MICROSHIELD® User's Manual, Grove Software, Inc. (2009).

[9] N. Odano, T. Miura and Y. Shindo, Experimental Assessment of Gamma-ray Shielding Transport Calculations by Monte Carlo Calculation Code and Point-kernel Code, Proc. of ICRS-8, Arlington, USA, Apr. 24-28, 1994, p. 1301. 